CARNETS DE Carnets de géographes

GÉOGRAPHES.

$1 \mid 2010$

Varia

\title{
Analyse de la perception et des fonctions des tourbières
}

L'exemple rhônalpin

Céline Sacca

C OpenEdition

Journals

Édition électronique

URL : http://journals.openedition.org/cdg/2299

DOI : $10.4000 /$ cdg.2299

ISSN : 2107-7266

Éditeur

UMR 245 - CESSMA

Référence électronique

Céline Sacca, "Analyse de la perception et des fonctions des tourbières », Carnets de géographes [En ligne], 1 | 2010, mis en ligne le 01 octobre 2010, consulté le 24 septembre 2020. URL : http:// journals.openedition.org/cdg/2299; DOI : https://doi.org/10.4000/cdg.2299

Ce document a été généré automatiquement le 24 septembre 2020.

\section{(c) (1) $\Theta$}

La revue Carnets de géographes est mise à disposition selon les termes de la Licence Creative Commons Attribution - Pas d'Utilisation Commerciale - Pas de Modification 4.0 International. 


\title{
Analyse de la perception et des fonctions des tourbières
}

\author{
L'exemple rhônalpin
}

\section{Céline Sacca}

1 Jusqu'à récemment l'étude des tourbières a essentiellement porté sur les aspects biophysiques. La richesse de leur biodiversité et l'originalité de leur fonctionnement hydrologique ont, en effet, été l'objet de très nombreux travaux scientifiques reléguant au second plan la dimension humaine de ces milieux pourtant bien réelle. Depuis quelques années, une évolution s'est toutefois dessinée sous l'impulsion notamment du Groupe d'Histoire des Zones Humides (GHZH) avec la volonté d'appréhender les tourbières au prisme des sociétés humaines leur conférant ainsi une dimension sociale et historique qui non seulement leur faisait défaut, mais autorise également la compréhension biophysique et la gestion patrimoniale de ces milieux palustres. Parallèlement l'intégration des fonctions des écosystèmes dans les stratégies de conservation a indéniablement renouvelé les critères pris en compte pour asseoir la préservation des zones humides. D'abord naturelles, les fonctions renvoient aujourd'hui aux nombreux rôles et services que la société assigne aux écosystèmes et qu'elle juge utile pour elle-même.

2 Situé à l'interface entre les tourbières et la société, ce travail a pour objet d'identifier et d'évaluer les fonctions des tourbières rhônalpines. Si à l'échelle régionale, le cadre physique est globalement favorable à la turfigenèse et présente une grande diversité de tourbières, l'aire d'étude résulte moins d'une réflexion géographique au sens convenu du terme que d'une conjoncture politique et scientifique propice. La région et les gestionnaires se sont, en effet, engagés dans une politique accrue de préservation qui a conduit à une connaissance relativement bonne des tourbières régionales grâce à l'inventaire réalisé par le Conservatoire Régional des Espaces Naturels (CREN). 623 sites ont été répertoriés pour une superficie estimée à 10000 ha soit $0,2 \%$ du territoire de la région. Il faut également souligner une dynamique de recherche importante encouragée par le lancement en 2003 du programme de recherche PETRA (Programme d'Étude des Tourbières RhônAlpine). 

des tourbieres rhonapines au sein de l'espace régiona à partir de méthodes statistiques et multicritères. L'analyse de la base de données du CREN a ainsi permis d'observer grâce à une analyse factorielle des correspondances, les rapprochements et les oppositions entre les caractéristiques des tourbières régionales. Le prolongement de cette analyse par une classification hiérarchique a quant à lui, fait apparaître une typologie des tourbières selon leur degré de ressemblance. Quant aux méthodes multicritères, elles ont permis de construire sur la base de plusieurs critères un profil fonctionnel pour chaque tourbière.

7 Ensuite et ce, afin de comprendre la façon dont se sont instituées les relations entre les tourbières et la société, le second volet du travail a porté sur les usages depuis le XIX ème siècle, période charnière du rapport Homme/milieu. L'approche privilégiée a été une approche diachronique conduite à partir de méthodes historiques (consultations d'archives, documents cadastraux, études toponymiques...). Deux échelles d'analyse ont été appréhendées : une échelle locale à travers un échantillon de sites et une échelle plus petite, en l'occurrence celle de la région, afin de conserver une vue d'ensemble. Les moyens d'investigation ont été variables selon le niveau d'analyse concerné.

Enfin, le troisième volet s'est intéressé à la portée socio-culturelle des tourbières souvent avancée mais rarement étudiée. Inscrites dans des territoires que les hommes se sont appropriés, les écosystèmes tourbeux mettent en relation différents acteurs qui ont développé à leur égard des rapports tout aussi différenciés. L'objectif était donc de restituer des visions plus variées qu'on ne le croit, de permettre le croisement de divers points de vue sur l'objet, d'éclairer la complexité des pratiques et des représentations et d'en révéler l'épaisseur. C'est donc une approche qualitative qui a été préconisée au travers d'entretiens semi-directifs.

$9 \quad$ Au terme de ce travail, les résultats invitent à relativiser les fonctions des tourbières et incitent à substituer à l'approche générale une approche plus différenciée dont les fils conducteurs devraient être la prise en compte de la capacité des tourbières à remplir les fonctions et la considération non plus de l'écosystème mais du milieu c'est-à-dire l'ensemble des interactions entre les hommes et les tourbières.

Fiche informative

Thèse disponible en ligne http://tel.archives-ouvertes.fr/tel-00399845/fr/ 


\section{Discipline}

Géographie

\section{Directeur}

\section{Bernard ETLICHER}

\section{Université}

Saint Etienne

Membres du jury thèse, soutenue le 12 juin 2009

- Jean-Paul AMAT, Professeur, Université de Paris IV, rapporteur

- Hervé CUBIZOLLE, Professeur, Université de Saint Étienne, examinateur

- Bernard ETLICHER, Professeur, Université de Saint Étienne, directeur

- Hervé GUMUCHIAN, Professeur émérite, Université de Grenoble I, rapporteur

- Francis MULLER, Directeur du pôle-relais tourbières de Besançon, invité

- Bertrand SAJALOLI, Maitre de conférence, Université d'Orléans, examinateur

\section{Situation professionnelle actuelle}

Ingénieur d'étude sur le projet PATRA août 2010 - février 2011 (patrimoine et trajectoires paysagères de la vallée de la Loire) au Centre de Recherche en Environnement et Aménagement

\section{Contact}

Celine.Sacca[at]univ-st-etienne.fr

INDEX

Thèmes : Carnets de soutenances 